\title{
Noncommutative uniform algebras
}

\author{
by \\ Mati Abel (Tartu) and Krzysztof Jarosz (Edwardsville, IL)
}

\begin{abstract}
We show that a real Banach algebra $A$ such that $\left\|a^{2}\right\|=\|a\|^{2}$ for $a \in A$ is a subalgebra of the algebra $C_{\mathbb{H}}(X)$ of continuous quaternion-valued functions on a compact set $X$.
\end{abstract}

1. Introduction. The well known Hirschfeld-Żelazko Theorem [5] (see also [7]) states that for a complex Banach algebra $A$ the condition

$$
\left\|a^{2}\right\|=\|a\|^{2} \quad \text { for } a \in A
$$

implies that (i) $A$ is commutative, and further that (ii) $A$ must be of a very specific form, namely it must be isometrically isomorphic to a uniformly closed subalgebra of $C_{\mathbb{C}}(X)$. We denote here by $C_{\mathbb{C}}(X)$ the complex Banach algebra of all continuous functions on a compact space $X$. The obvious question about the validity of the same conclusion for real Banach algebras is immediately dismissed with an obvious counterexample: the non-commutative algebra $\mathbb{H}$ of quaternions. However it turns out that the second part (ii) above is essentially also true in the real case. We show that the condition (1.1) implies, for a real Banach algebra $A$, that $A$ is isometrically isomorphic to a subalgebra of $C_{\mathbb{H}}(X)$, the algebra of continuous quaternion-valued functions on a compact space $X$. That result is in fact a consequence of a theorem by Aupetit and Zemánek [1]. We will also present several related results and corollaries valid for real and complex Banach and topological algebras. To simplify the notation we assume that the algebras under consideration have units, however, as in the case of the Hirschfeld-Żelazko Theorem, the analogous results can be stated for non-unital algebras as one can formally add a unit to such an algebra.

We use standard notation, as can be found for example in [6]. For a real Banach algebra $A$ with a unit $e$ we denote by $A^{-1}$ the set of invertible elements of $A$; for $a \in A$ we define the real and complex spectra and the

2000 Mathematics Subject Classification: Primary 46H15; Secondary 47L55.

Key words and phrases: Banach algebra, uniform algebra, function algebra.

Research partially supported by a grant from the National Research Council. 
corresponding spectral radii by well known formulas:

$$
\begin{aligned}
\sigma_{\mathbb{R}}(a) & =\left\{s \in \mathbb{R}: a-s e \notin A^{-1}\right\}, \\
\sigma_{\mathbb{C}}(a) & =\left\{s+i t \in \mathbb{C}:(a-s e)^{2}+t^{2} e \notin A^{-1}\right\}, \\
\varrho_{\mathbb{R}}(a) & =\sup \left\{|\lambda|: \lambda \in \sigma_{\mathbb{R}}(a)\right\}, \\
\varrho_{\mathbb{C}}(a) & =\sup \left\{|\lambda|: \lambda \in \sigma_{\mathbb{C}}(a)\right\},
\end{aligned}
$$

where we put $\varrho_{\mathbb{R}}(a)=0$ if the set $\sigma_{\mathbb{R}}(a)$ is empty. It is well known [6] that $\sigma_{\mathbb{C}}(a)=\left\{\lambda \in \mathbb{C}: a-\lambda e \notin A_{\mathbb{C}}^{-1}\right\}$, where $A_{\mathbb{C}}$ is the complexification of $A$, and that $\varrho_{\mathbb{C}}(a)=\lim \sqrt[n]{\left\|a^{n}\right\|}$.

\section{The results}

TheOrem 1. Assume $A$ is a real Banach algebra such that $\|a\| \leq C \varrho_{\mathbb{C}}(a)$ for some positive constant $C$ and all $a \in A$. Then $A$ is isomorphic to a uniformly closed subalgebra of $C_{\mathbb{H}}(X)$ for some compact space $X$.

To prove the theorem we need the following special case of the main result of [1].

Theorem 2 (Aupetit-Zemánek). Let $A$ be a real Banach algebra with unit. If there is a constant $\beta$ such that $\varrho_{\mathbb{C}}(a b) \leq \beta \varrho_{\mathbb{C}}(a) \varrho_{\mathbb{C}}(b)$ for all $a, b$ in $A$ then for every irreducible representation $\pi: A \rightarrow L(E)$, the algebra $\pi(A)$ is isomorphic to its commutant $C_{\pi}$ in the algebra $L(E)$ of all linear transformations on $E$.

Proof of Theorem 1. It is clear that our condition $\|a\| \leq C \varrho_{\mathbb{C}}(a)$ implies that $\varrho_{\mathbb{C}}(a b) \leq \gamma \varrho_{\mathbb{C}}(a) \varrho_{\mathbb{C}}(b)$, with $\gamma=C^{2}$. Since the commutant $C_{\pi}$ is a normed real division algebra $([3$, p. 127$])$ it is isomorphic to $\mathbb{R}, \mathbb{C}$, or $\mathbb{H}$. Hence by Theorem 2 any irreducible representation of $A$ in an algebra of linear maps on a Banach space is equivalent to a representation into $\mathbb{H}$; let $X$ be the set of all such equivalence classes of irreducible representations of $A$. For $x \in X$ and $a \in A$ put $J(a)(x)=x(a) \in \mathbb{H}$. The map $J$ is an isomorphism of $A$ into the algebra of $\mathbb{H}$-valued functions on $X$. One could introduce a compact topology on $X$ making all functions $J(a)$ continuous by referring to the structure space topology on the space of all irreducible representations; we would like to give a more direct and elementary argument.

Let $\tau$ be the topology on $X$ generated by $J(a), a \in A$, that is, the weakest topology such that all the functions $J(a), a \in A$, are continuous. By the definition of $X$, for any two distinct points $x_{1}, x_{2} \in X$ there is an $a \in A$ such that $J(a)\left(x_{1}\right) \neq J(a)\left(x_{2}\right)$, so $\tau$ is Hausdorff. By [4, Ex. 1.5.E(a), p. 72] a topological Hausdorff space is completely regular if and only if its topology is generated by a family of real-valued functions. Since an $\mathbb{H}$-valued function is continuous if and only if all four of its coordinates are, 
$X$ is completely regular and consequently has a maximum compactification $\beta X$ ([4]). Assume $\beta X \backslash X$ is not empty and let $x_{0} \in \beta X \backslash X$. The operator $A \ni a \mapsto(J(a))\left(x_{0}\right) \in \mathbb{H}$ is an irreducible representation on $A$ so $x_{0} \in X$; this contradiction shows that $X$ is compact.

To show that $J$ is injective we need to show that $A$ is semisimple. Fix $0 \neq a_{0} \in A$ and let $A_{0}$ be the closed subalgebra of $A$ generated by all the elements of the form $q\left(a_{0}\right)$, where $q$ is a rational function with real coefficients and with poles outside $\sigma_{\mathbb{C}}\left(a_{0}\right)$. Notice that $A_{0}$ is a commutative Banach algebra such that, by our assumption, the spectrum of each nonzero element is not trivial, so $\operatorname{rad} A_{0}=\{0\}$. If $b \in A^{-1} \cap A_{0}$ then $b^{-1}$, being given by a rational function, is in $A_{0}$; that means that $A^{-1} \cap A_{0}=A_{0}^{-1}$. Hence by [8, p. 476], $A_{0} \cap \operatorname{rad} A \subset \operatorname{rad} A_{0}=\{0\}$, so $a_{0} \notin \operatorname{rad} A$, and since $a_{0}$ was arbitrary, $A$ is semisimple.

Corollary 1. Assume $A$ is a real Banach algebra such that $\|a\|^{2} \leq$ $C\left\|a^{2}\right\|$ for some positive constant $C$ and all $a \in A$. Then $A$ is isomorphic to a uniformly closed subalgebra of $C_{\mathbb{H}}(X)$ for some compact set $X$; if $C=1$ then the isomorphism is also an isometry.

Corollary 2. Assume $A$ is a real m-convex Hausdorff algebra with the topology given by a family $\left\{p_{\alpha}: \alpha \in \Lambda\right\}$ of m-convex seminorms. If for every $\alpha \in \Lambda$ there is a constant $C_{\alpha}$ such that $\left(p_{\alpha}(a)\right)^{2} \leq C_{\alpha} p_{\alpha}\left(a^{2}\right)$ for all $a \in A$ then $A$ is a subalgebra of $C_{\mathbb{H}}(X)$ for some Hausdorff space $X$.

Proof. Fix $\alpha \in \Lambda$ and let $A_{\alpha}$ be the completion of the normed algebra $A / \operatorname{ker} p_{\alpha}$; we denote by $q_{\alpha}$ the norm on $A_{\alpha}$ generated by $p_{\alpha}$. Since $p_{\alpha}$ is continuous we have $\left(q_{\alpha}(f)\right)^{2} \leq C_{\alpha} q_{\alpha}\left(f^{2}\right)$ for all $f \in A_{\alpha}$. By the previous corollary, $A_{\alpha}$ is a uniformly closed subalgebra of $C_{\mathbb{H}}\left(X_{\alpha}\right)$ for some compact space $X_{\alpha}$. We now obtain the promised representation of $A$ as a subalgebra of $C_{\mathbb{H}}(X)$ by taking the projective limit of the algebras $A_{\alpha} \subset C_{\mathbb{H}}\left(X_{\alpha}\right)$.

TheORem 3. Assume $A$ is a real Banach algebra such that $\|a\| \leq C \varrho_{\mathbb{R}}(a)$ for some positive constant $C$ and all $a \in A$. Then $A$ is isomorphic to the algebra $C_{\mathbb{R}}(X)$ for some compact space $X$.

Proof. Since $\|a\| \leq C \varrho_{\mathbb{R}}(a) \leq C \varrho_{\mathbb{C}}(a)$, it follows from Theorem 1 that $A \subset C_{\mathbb{H}}(X)$ for some compact space $X$. We show that for any $f \in A$ we actually have $f \in C_{\mathbb{R}}(X)$. Let $A_{f}$ be the closed subalgebra of $A$ generated by $f$; since $A_{f}$ is commutative we have $A_{f} \subset C_{\mathbb{C}}(X)$. For any $g \in A_{f}$ we have $\sigma_{\mathbb{R}}(g) \subset\{\operatorname{Re} g(x): x \in X\}$ and consequently by our assumption $\|g\| \leq C\|\operatorname{Re} g\|$. Hence for any sequence $\left(g_{n}\right)$ in $A_{f}$ if $\left(\operatorname{Re} g_{n}\right)$ is a Cauchy sequence in $C_{\mathbb{R}}(X)$, then $\left(g_{n}\right)$ is a Cauchy sequence in $A_{f} \subset C_{\mathbb{C}}(X)$, so $\operatorname{Re} A$ is uniformly closed. By [6, Th. 2.3.3] $A_{f}=C(X, \tau)$ for some topological involution $\tau: X \rightarrow X$. Assume there is a point $x_{0}$ in $X$ such that $\tau\left(x_{0}\right) \neq x_{0}$ 
and let $U$ be a neighborhood of $x_{0}$ such that $\tau(U) \cap U=\emptyset$. Let $h_{0} \neq 0$ be a real-valued continuous function with support in $U$ and define

$$
h_{1}(x)= \begin{cases}i h_{0}(x) & \text { for } x \in U, \\ -i h_{0}\left(\tau^{-1}(x)\right) & \text { for } x \in \tau(U), \\ 0 & \text { for } x \in X \backslash(U \cup \tau(U)) .\end{cases}
$$

We have $h_{1} \in C(X, \tau)=A_{f}$ but $\varrho_{\mathbb{R}}\left(h_{1}\right)=0$, which contradicts our assumption. Hence $\tau=\operatorname{id}_{X}$ and consequently $f$ is real-valued.

Corollary 3. If $K$ is a compact subset of the complex plane not fully contained in the real line then for any $C>0$ there is a polynomial $p$ with real coefficients such that

$$
\sup \{|\operatorname{Re}(p(z))|: z \in K\}<1 \quad \text { while } \sup \{|p(z)|: z \in K\}>C .
$$

Proof. Let $A$ be the uniform closure of the subalgebra of $C_{\mathbb{R}}(K)$ consisting of all polynomials with real coefficients. Let $z_{0} \in K \backslash \mathbb{R}$. Since $\overline{f\left(z_{0}\right)}=$ $f\left(\bar{z}_{0}\right)$ for all $f \in A$ it follows that $A$ is not of the form $C_{\mathbb{R}}(X)$, for any compact space $X$. By the last theorem for any $C>0$ there is $f \in A$ such that

$\sup \{|\operatorname{Re} f(z)|: z \in K\}=\varrho_{\mathbb{R}}(f)<1 \quad$ while $\sup \{|f(z)|: z \in K\}=\varrho_{\mathbb{C}}(f)>C$, and since the polynomials with real coefficients are dense in $A$ we get the corollary.

Corollary 4. If $A$ is a real algebra then there is a constant $C$ such that

$$
\varrho_{\mathbb{C}}(a) \leq C \varrho_{\mathbb{R}}(a) \quad \text { for all } a \in A
$$

if and only if

$$
\sigma_{\mathbb{R}}(a)=\sigma_{\mathbb{C}}(a) \quad \text { for all } a \in A .
$$

Proof. One implication is obvious. To prove the other assume there is $a_{0} \in A$ such that $K=\sigma_{\mathbb{C}}\left(a_{0}\right) \nsubseteq \mathbb{R}$ and let $p$ be a polynomial with real coefficients given by the previous corollary. We have $\varrho_{\mathbb{C}}\left(p\left(a_{0}\right)\right)=\sup \{|p(z)|$ : $z \in K\}>C$ while $\varrho_{\mathbb{R}}\left(p\left(a_{0}\right)\right) \leq \sup \{|\operatorname{Re} p(z)|: z \in K\}<1$ contrary to our assumptions.

The above results show that certain inequalities between the real spectrum, the complex spectrum, and the norm in a topological algebra can provide very strong conclusions concerning the structure of the algebra. Another example of a similar situation is provided by the Kulkarni Theorem [6]: if $\|a\|^{2} \leq\left\|a^{2}+b^{2}\right\|$ for all $a, b$ with $a b=b a$ in a real algebra $A$, then the algebra is commutative. It is natural to ask what conclusion could be obtained from the assumption that $a b$ is related by a specific inequality to 
$b a$, for arbitrary elements of a noncommutative topological algebra. Some results in this direction can be obtained by using the well known technique (see e.g. [2, p. 330]) of analyzing the entire function

$$
\lambda \stackrel{\varphi}{\longmapsto} \exp (\lambda a) b \exp (-\lambda a) \in A .
$$

For example if $A$ is a complex m-convex Hausdorff algebra with the topology given by a family $\left\{p_{\alpha}: \alpha \in \Lambda\right\}$ of m-convex seminorms, and if for any $\alpha \in \Lambda$ there is a constant $C_{\alpha}$ such that

$$
p_{\alpha}(a b) \leq C_{\alpha} p_{\alpha}(b a) \quad \text { for all } a, b \in A
$$

then $A$ is commutative. Indeed, in this case for any $\lambda$ and $\alpha$ we have $p_{\alpha}(\varphi(\lambda)) \leq C_{\alpha} p_{\alpha}(b)<\infty$ so the function

$$
\varphi(\lambda)=e+(a b-b a) \lambda+(\ldots) \lambda^{2}+\ldots
$$

is bounded in all the algebras $A_{\alpha}, \alpha \in \Lambda$, where $A_{\alpha}$ is the completion of the normed algebra $A / \operatorname{ker} p_{\alpha}$. Since the continuous linear functionals on $A_{\alpha}$ separate the points of this algebra, we get $a b-b a \in \operatorname{ker} p_{\alpha}$, and consequently $a b=b a$. The are two crucial elements in the proof: we consider the complex case, and there are enough continuous linear functionals to separate the points. Hence basically the same proof works for the complex ample $p$-algebras or Mackey complete locally normed algebras. However it is an open problem whether a similar result can be obtained for complex topological algebras without continuous functionals, for example for the class of $p$-algebras, or for real algebras.

Below we state another problem asking if an almost commutative multiplication must be close to a commutative one.

Conjecture 1. Assume $A$ is a Banach algebra, $\varepsilon>0$ and that

$$
\|a b-b a\| \leq \varepsilon\|a\|\|b\|, \quad a, b \in A .
$$

Then there is a commutative multiplication $\times$ on $A$ with $\|a b-a \times b\| \leq$ $\varepsilon^{\prime}\|a\|\|b\|$ where $\varepsilon^{\prime}=\varepsilon^{\prime}(\varepsilon) \rightarrow 0$ as $\varepsilon \rightarrow 0$.

Notice that the condition (2.2) does not imply that the original multiplication is commutative. Indeed, if we take an arbitrary Banach algebra $A$ we can define a new multiplication - on the Banach space $A$ by $a \cdot b=\frac{\varepsilon}{2} a b$. The Banach algebra $(A, \cdot)$ satisfies $(2.2)$ but need not be commutative. The situation does not change if we add the assumption that $A$ has a unit-we can add it artificially to $(A, \cdot)$. This example does not however contradict the conjecture since $\cdot$ is close to the zero multiplication.

The authors would like to thank S. H. Kulkarni for a number of valuable comments on the first draft of the paper. 


\section{References}

[1] B. Aupetit and J. Zemánek, On the real spectral radius in real Banach algebras, Bull. Acad. Polon. Sci. Sér. Sci. Math. Astronom. Phys. 26 (1978), 969-973.

[2] V. K. Balachandran, Topological Algebras, North-Holland Math. Stud. 185, NorthHolland, 2000.

[3] F. F. Bonsall and J. Duncan, Complete Normed Algebras, Springer, Berlin, 1973.

[4] R. Engelking, General Topology, PWN, Warszawa, 1977.

[5] R. A. Hirschfeld and W. Żelazko, On spectral norm Banach algebras, Bull. Acad. Polon. Sci. Sér. Sci. Math. Astronom. Phys. 16 (1968), 195-199.

[6] S. H. Kulkarni and B. V. Limaye, Real Function Algebras, Monographs and Textbooks in Pure and Appl. Math. 168, Dekker, 1992.

[7] C. Le Page, Sur quelques conditions entraînant la commutativité dans les algèbres de Banach, C. R. Acad. Sci. Paris Sér. A-B 265 (1967), A235-A237.

[8] T. Palmer, Banach Algebras and the General Theory of *-Algebras, Vol. I, Algebras and Banach Algebras, Encyclopedia Math. Appl. 49, Cambridge Univ. Press, Cambridge, 1994.

Department of Mathematics

University of Tartu

51014 Tartu, Estonia

E-mail: abel@math.ut.ee
Department of Mathematics Southern Illinois University Edwardsville Edwardsville, IL 62026, U.S.A. E-mail: kjarosz@siue.edu http://www.siue.edu/ ${ }^{\sim}$ kjarosz

Received July 22, 2002

Revised version September 8, 2003 